\title{
Human kidney organoids produce functional renin
}

\author{
Anusha S. Shankar ${ }^{1,7}$, Zhaoyu Du ${ }^{1}$, Hector Tejeda Mora ${ }^{1}$, Thierry P.P. van den Bosch², \\ OPEN \\ Sander S. Korevaar ${ }^{1}$, Ingrid M. Van den Berg-Garrelds ${ }^{3}$, Eric Bindels ${ }^{4}$, Carmen Lopez-Iglesias ${ }^{5}$, \\ Marian C. Clahsen-van Groningen², Joost Gribnau ${ }^{6}$, Carla C. Baan ${ }^{1}$, A.H. Jan Danser ${ }^{3}$, Ewout J. Hoorn ${ }^{1}$ \\ and Martin J. Hoogduijn
}

${ }^{1}$ Department of Internal Medicine, Division of Nephrology and Transplantation, Erasmus MC, University Medical Center, Rotterdam, The Netherlands; ${ }^{2}$ Department of Pathology, Erasmus MC, University Medical Center, Rotterdam, The Netherlands; ${ }^{3}$ Department of Internal Medicine, Division of Pharmacology and Vascular Medicine, Erasmus MC, University Medical Center, Rotterdam, The Netherlands; ${ }^{4}$ Department of Haematology, Erasmus MC Cancer Institute, Rotterdam, The Netherlands; ${ }^{5}$ Maastricht Multimodal Molecular Imaging Institute, Maastricht University, Maastricht, The Netherlands; and ${ }^{6}$ Department of Developmental Biology and iPS Core Facility, Erasmus MC, University Medical Center, Rotterdam, The Netherlands

Renin production by the kidney is of vital importance for salt, volume, and blood pressure homeostasis. The lack of human models hampers investigation into the regulation of renin and its relevance for kidney physiology. To develop such a model, we used human induced pluripotent stem cell-derived kidney organoids to study the role of renin and the renin-angiotensin system in the kidney. Extensive characterization of the kidney organoids revealed kidneyspecific cell populations consisting of podocytes, proximal and distal tubular cells, stromal cells and endothelial cells. We examined the presence of various components of the renin-angiotensin system such as angiotensin II receptors, angiotensinogen, and angiotensin-converting enzymes 1 and 2 . We identified by single-cell sequencing, immunohistochemistry, and functional assays that cyclic AMP stimulation induces a subset of pericytes to increase the synthesis and secretion of enzymatically active renin. Renin production by the organoids was responsive to regulation by parathyroid hormone. Subcutaneously implanted kidney organoids in immunodeficient IL2Ry'/Rag2 $^{-/-}$mice were successfully vascularized, maintained tubular and glomerular structures, and retained capacity to produce renin two months after implantation. Thus, our results demonstrate that kidney organoids express renin and provide insights into the endocrine potential of human kidney organoids, which is important for regenerative medicine in the context of the endocrine system.

Kidney International (2021) 99, 134-147; https://doi.org/10.1016/ j.kint.2020.08.008

KEYWORDS: cyclic AMP; induced pluripotent stem cells; kidney organoids; parathyroid hormone; pericyte; renin-angiotensin system

Copyright (c) 2020, International Society of Nephrology. Published by Elsevier Inc. This is an open access article under the CC BY license (http:// creativecommons.org/licenses/by/4.0/).

Correspondence: Anusha Shweta Shankar, Department of Internal Medicine, Erasmus MC, University Medical Center, Postbox 2040, 3000 CA, Rotterdam, The Netherlands. E-mail: a.shankar@erasmusmc.nl

${ }^{7}$ Lead author.

Received 3 April 2020; revised 29 July 2020; accepted 10 August 2020; published online 9 September 2020

\section{Translational Statement}

The advancement of knowledge and technology in the organoid field has allowed the generation of structurally complex and functional kidney organoids. We demonstrate that renin, a key factor in blood pressure regulation produced by the kidney, is produced and secreted by kidney organoids. This work demonstrates that kidney organoids can be used to test the effect of novel drugs on the renin-angiotensin system and contributes to the field of regenerative medicine to replace the hormonal system of injured kidneys. 
the juxtaglomerular apparatus at the terminal part of the afferent arterioles. The exact mechanisms regulating renin expression during development are poorly understood. This is partly due to the lack of suitable human in vitro models to study the RAS, as juxtaglomerular cells are rare and rapidly lose their ability to produce renin in culture. ${ }^{7-9}$ Kidney organoids may represent a model to study the RAS in a human system.

As current kidney organoids resemble the early developmental stages of the kidney, they could be used for examining the unique patterning of renin-producing cells and their contribution to the growth and differentiation of the kidney. Eventually kidney organoids with a functional endocrine system may be developed that have the capacity to normalize hormonal imbalances in patients with kidney failure. Our hypothesis asserts the existence of a functional RAS in human iPSC-derived kidney organoids. Hence, we investigated the regulation of renin production in kidney organoids and characterized the renin-producing cells using imaging techniques and single-cell RNA sequencing (scRNAseq) analyses. Finally, we showed that renin-producing cells in kidney organoids maintain their hormonal production after implantation.

\section{RESULTS}

\section{Human iPSC-derived kidney organoids contain renal structures and display tubular transport}

Kidney organoids were differentiated from human iPSCs through a protocol involving 7 days of monolayer culture followed by 18 days of 3-dimensional culture and the timely addition of CHIR99021 and fibroblast growth factor 9 (Figure 1a). At day 25, complex structures were visible by bright-field microscopy (Figure $1 \mathrm{~b}$ and $\mathrm{c}$ ). Intermediate mesoderm markers such as OSR1 and PAX2 showed a 90and 3000-fold mRNA upregulation compared with iPSCs (Figure 1d). Wilms tumor 1 (WT1) and homeobox protein Hox-D11 (HOXD11), transcription factors crucial for the nephron lineage, increased 9000- and 20,000-fold, whereas glial-derived neurotrophic factor (GDNF), a marker for the ureteric epithelium, increased 18 -fold after differentiation. The expression of pluripotent markers nanog homeobox (NANOG), C-MYC, octamer-binding transcription factor 4 (OCT-4), and SOX2 was downregulated, whereas expression of REX1 and Krüppel-like factor 4 (KLF4) remained unchanged (Figure 1d). At day 25, immunohistochemistry revealed that $\mathrm{WT}_{1}^{+}$, Villin- $1^{+}$, E-cadherin $\left(\mathrm{ECAD}^{+}\right)$, and $\mathrm{CD} 31^{+} / \mathrm{CD} 34^{+}$cells were present, suggesting the emergence of glomeruli, proximal tubuli, distal tubuli, ureteric epithelium and endothelial cells, respectively (Figure le and f). COL1A1 and platelet-derived growth factor receptor A $(P D G F R \alpha)$ marked the basement membranes and renal interstitium (Figure 1f). Electron microcopy showed the presence of proximal tubular structures containing a single layer of polarized epithelial cells connected by tight junctions on day 25 (Figure $1 \mathrm{~g}$ ). The tubuli contained an open lumen with brush border microvilli along with mitochondria, lysosomes, and small apical endocytic vacuoles. Sodium transporters sodium-proton-exchanger subtype 3 (NHE3) and sodium-potassium-chloride transporter 2 (NKCC2) were observed at the apical side of tubular structures, suggesting a loop of Henle population (Figure 1h). We observed upregulation of mRNA of transporters for small organic anions, OAT1 and OAT3, megalin, and cubilin (Figure 1i). The functionality of organic anion transporter 1 (OAT1) and OAT3 was demonstrated by the active transport of the organic anion fluorescein at $37{ }^{\circ} \mathrm{C}$ but not at $4{ }^{\circ} \mathrm{C}$ into the lumen of tubuli (Figure 1j). To profile the various cell populations in the kidney organoids, we performed scRNAseq on a day- 25 kidney organoid (Figure 1k). Clustering confirmed the presence of podocytes (22.1\% of the cells), proximal (22.3\%) and distal tubular cells/loop of Henle (2.3\%), endothelial cells $(0.5 \%)$, pericytes $(8.6 \%)$, and stromal cells $(3.4 \%)$. Populations of neural cells (20.5\%) and cell-cycling cells (20\%) were also found. Expression levels of marker genes within each cluster and the proportion of cells expressing a gene were visualized in a dot plot (Figure 11). These data demonstrate that iPSC-derived organoids contain the major cell types of the kidney. We then continued studying the presence of the RAS in the kidney organoids.

\section{Components of the RAS are found in kidney organoids}

In the kidney organoids, renin mRNA increased 146-fold during differentiation of iPSC to intermediate mesoderm at day 7 (Figure 2a). Hereafter, the expression of renin in the organoids at day 25 decreased 45 -fold. Expression of AGTR1 and AGTR2 mRNA was 80-fold and 26,000-fold increased, respectively, compared with undifferentiated iPSCs (Figure 2b). We also detected a 166-fold increase in angiotensinogen (AGT) mRNA in the kidney organoids (Figure 2c). ACE mRNA was detected throughout organoid differentiation (Figure 2c). Expression of renin was most pronounced in a small population of pericytes (Figure 2d). In this cluster, pericyte markers such as NG2, MEIS1, MEIS2, $P D G F R \alpha, P D G F R \beta, C O L 1 A 1$, and COL3A1 were highly expressed. Unexpectedly, this compartment along with the stromal cluster exhibited the highest expression of the genes AGTR1 and AGTR2, whereas the tubular cells, podocytes, and endothelial cells did not express these receptors. AGT mRNA was expressed in proximal and distal tubular cells; however, AGT protein was absent in the supernatant of the kidney organoids (data not shown, $\mathrm{n}=8$ ). ACE was expressed by a low number of cells in the podocyte, distal tubular, and endothelial cell clusters. scRNAseq analysis revealed components of the alternative RAS, including protease prolyl carboxypeptidase $(P R C P)$ and prolyl endopeptidase (PREP) (Figure 2d). Glomerular and proximal tubular localization of alternative RAS components membrane metalloendopeptidase (MME) and angiotensin-converting enzyme 2 (ACE2) was confirmed with immunohistochemistry in day 25 organoids (Figure 2e), as shown recently by Monteil et al. ${ }^{10}$

To examine the influence of the RAS components on the development of kidney organoids, we added $500 \mathrm{nM}$ angiotensin II to kidney organoid cultures from day 12 onward in 
a

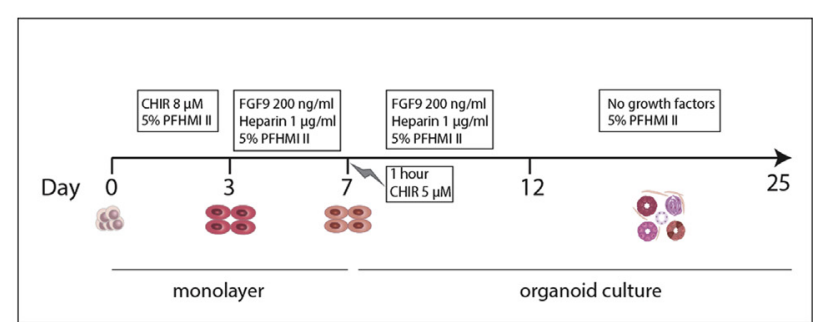

b

Day 1

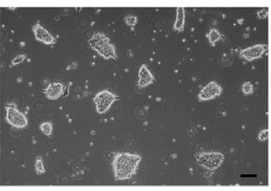

Day 7

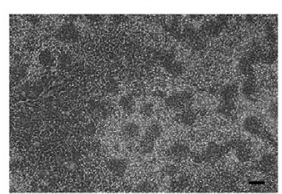

Day 25

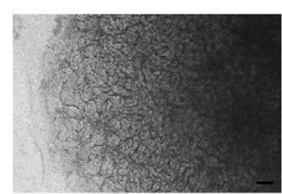

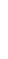
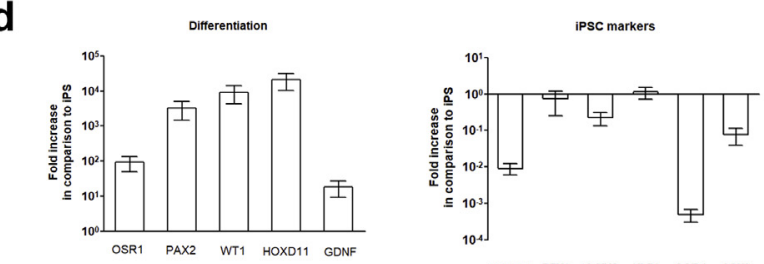

NANOG REX1 C-MYC KLFA OCTA SOX

e
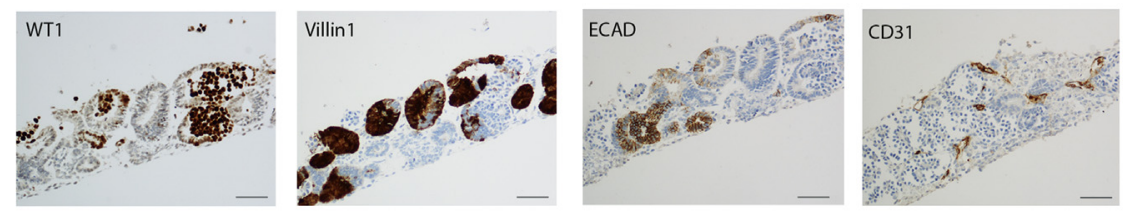

f
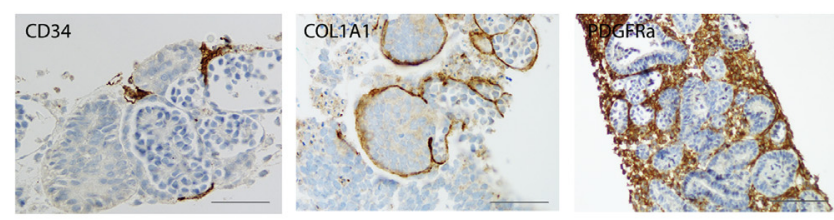

g
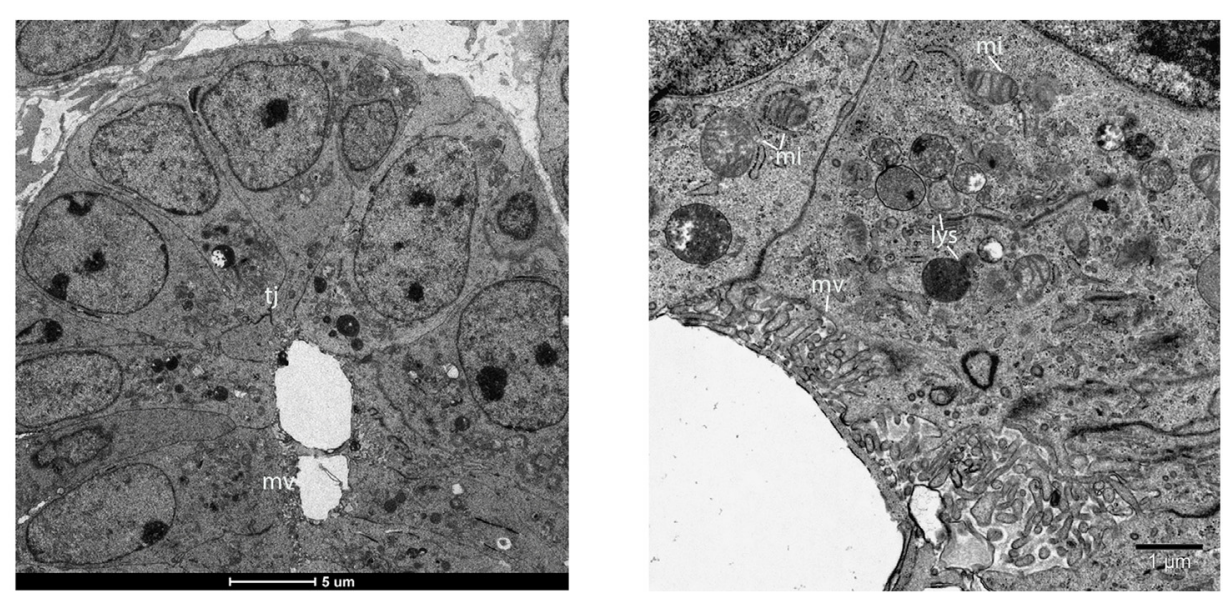

Figure 1 | Human iPSC-derived kidney organoids contain essential renal structures and display tubular transport. (a) A schematic overview of the kidney organoid culture. (b) Representative bright-field images of different time points during kidney organoid culture. Bars $=100 \mu \mathrm{m}$. (c) Image of a whole organoid at day 25. Bar $=500 \mu \mathrm{m}$. (d) Gene expression analysis of intermediate mesoderm, kidney differentiation markers, and pluripotency markers. Plots depict gene expression changes relative to undifferentiated iPSC. Data are represented as mean \pm SEM ( $n=4$ from 1 experiment). (e) Immunohistochemical analysis for markers of glomerular cells (WT1), proximal tubular cells (Villin-1), distal tubular cells (ECAD) and endothelial cells $\left(\mathrm{CD} 31^{+}\right)$. Bars $=100 \mu \mathrm{m}$. (f) Immunohistochemical analysis for markers of endothelial cells (CD34), basement membrane (COL1A1), and renal interstitium (PDGFR $\alpha$ ). Bars $=100 \mu \mathrm{m}$. (g) Electron microscopic analysis for the presence of tubular structures. Tj, tight junctions; mv, microvilli; mi, mitochondria; lys, lysosomes; sav, small apical endocytic vacuoles. (Continued) 
h
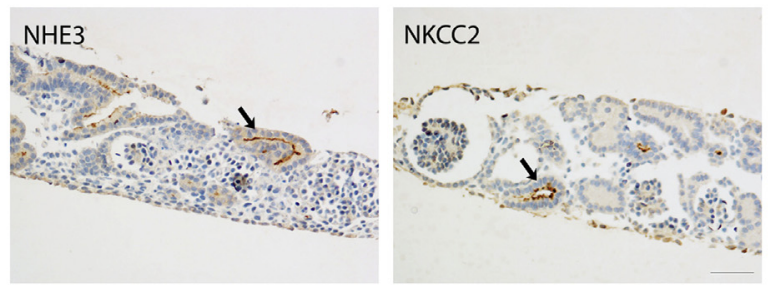

j
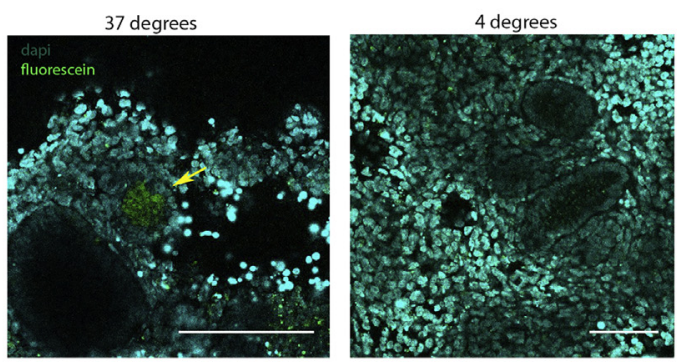

i

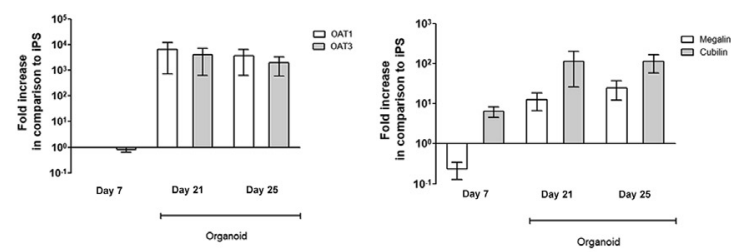

k

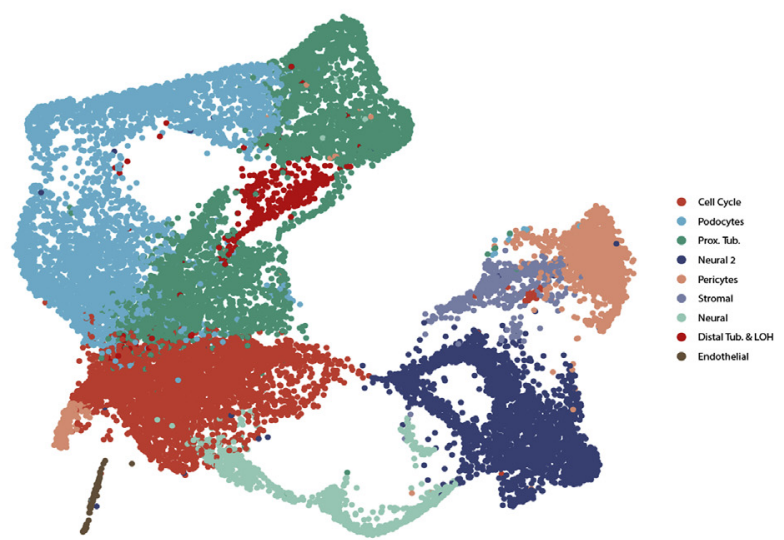

I

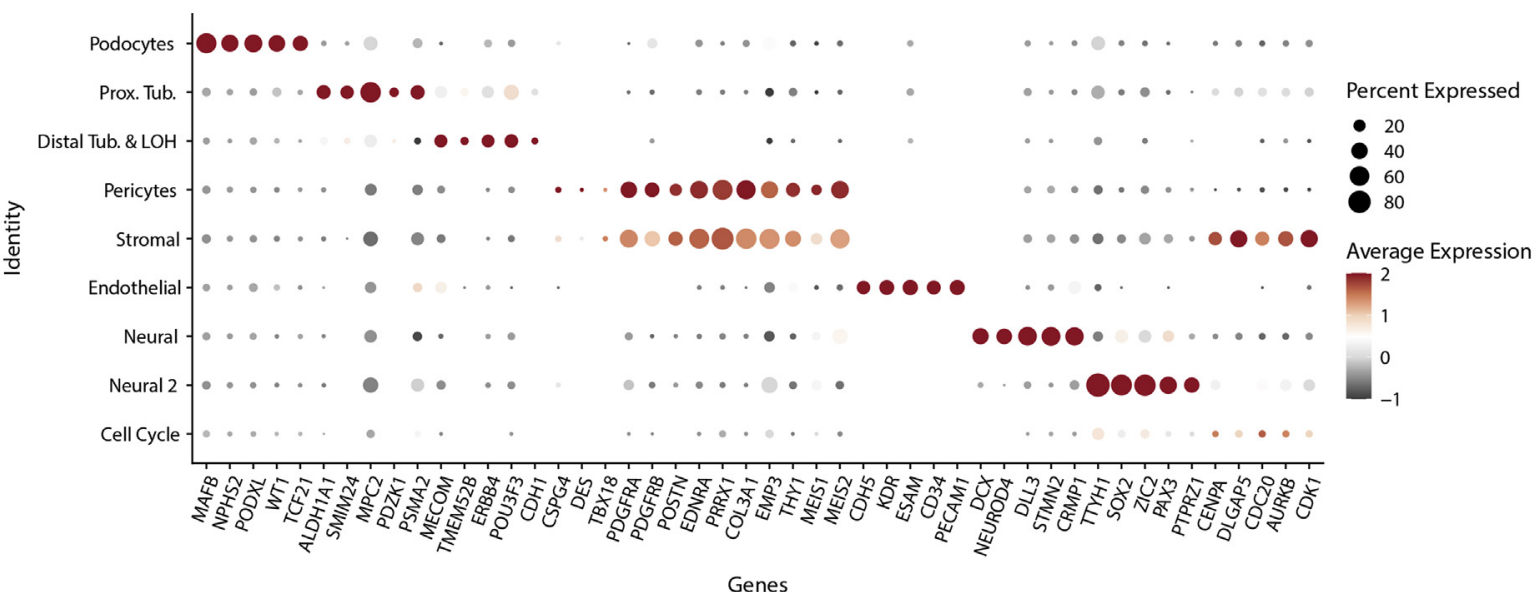

Figure 1| (Continued) (h) Immunohistochemistry for markers of the proximal tubuli (NHE3) and the loop of Henle (NKCC2) in the kidney organoid (as indicated by black arrows). Bars $=100 \mu \mathrm{m}$. (i) Gene expression analysis of various kidney transporters (OAT1, OAT3, megalin, and cubilin) over the course of the organoid culture. Plots depict gene expression changes relative to undifferentiated iPSC. Data are represented as mean \pm SEM ( $n=4$ from 1 experiment). (j) Fluorescein uptake assay showing uptake in lumen of tubuli (fluorescein, green; 4',6-diamidino-2-phenylindole [DAPI], blue). Bars $=50 \mu \mathrm{m}$. The left panel shows fluorescein uptake at $37^{\circ} \mathrm{C}$ for 1 hour (yellow arrow). The right panels shows the negative control at $4{ }^{\circ} \mathrm{C}$. (k) Uniform manifold approximation and projection plot displaying 9 distinct clusters of cell populations found in the kidney organoid. Populations have distinct colors to indicate identity. Prox. Tub., proximal tubule; Distal Tub. \& LOH, distal tubule and loop of Henle. (I) Dot plot visualization of representative marker genes per cluster of cells. ECAD, E-cadherin; iPSC, induced pluripotent stem cells; PDGFR $\alpha$, platelet-derived growth factor receptor A; SEM, standard error of the mean. To optimize viewing of this image, please see the online version of this article at www.kidney-international.org.

the presence or absence of AGTR1 antagonist losartan. We also added the direct renin inhibitor aliskiren throughout the culture period. Neither losartan nor aliskiren exerted any effect (Supplementary Figure S1). The expression of RAS components was not affected in angiotensin II-treated organoids, apart from a small increase in expression of AGTR1 (Supplementary Figure S1A). Angiotensin II drives fibrotic processes in the kidney, ${ }^{11}$ but we found no apparent effect of angiotensin II on fibrotic pathways in the kidney organoids (Supplementary Figure S1B). Gene and protein expression of WT1, Villin-1, and ECAD remained stable in all conditions (Supplementary Figure S1C and D) and profibrotic COL1A1 did not change when the patient was treated with angiotensin II (Supplementary Figure S1D). 


\section{Forskolin stimulates renin production in kidney organoids} There was a significant 305-fold increase in renin mRNA in forskolin-treated organoids (Figure 3a). In undifferentiated iPSCs, forskolin did not upregulate renin mRNA (Figure 3b). scRNAseq on a forskolin-stimulated organoid revealed that a significant subset of pericytes in the forskolin-treated organoid expressed renin (Figure $3 \mathrm{c}$ and $\mathrm{d}$ ). To obtain a closer perspective on the identity of these pericytes, we examined our previous transcriptome data in which reninomas and murine developing kidney were analyzed for their reninrelated gene signature $^{12}$ and identified core genes that constituted the gene profile of renin-expressing cells. Some of these genes were highly expressed in pericytes of the kidney organoid, including VIM, PDGFR $\beta$, and CXCL12 (Figure 3e). Interestingly, in our scRNAseq data analysis we noticed that a relatively low level of renin expression also emerged in the proximal tubular and-to a lesser extent-in the distal tubular compartment. The increase in renin mRNA was visible at the protein level, as forskolin stimulation caused a drastic increase in renin positivity (Figure $3 f$ ). We observed a diffuse distribution of renin-positive cells that expressed renin in a granular pattern (Figure 3f). Immunogold labeling of renin visualized cells mainly at the periphery of the organoids treated with forskolin, which may be classified as pericytes based on previous literature (Figure $3 \mathrm{~g}$ and h)., ${ }^{73}$ Multivesicular bodies from the late endocytic/lysosomal pathway could be observed that may represent secretory lysosomes that release their content upon trigger ${ }^{14}$ (Figure $3 \mathrm{~g}$ ). Double immunofluorescence of the pericyte marker PDGFR $\alpha$ and renin revealed colocalization of both markers (Figure 3i). Consecutive stainings for renin and the glomerular marker $\mathrm{WT}^{+}{ }^{+}$showed close proximity of the 2 proteins (Figure $3 \mathbf{j}$ ). In a week-13 human fetal kidney, renin-producing cells surround the glomeruli in a similar fashion (Figure $3 \mathrm{k}$ ). Next we demonstrated renin enzymatic activity in medium of organoids by the generation of angiotensin I, both before and after prorenin conversion to renin (Figure 31). Aliskiren blocked more than $99 \%$ of the angiotensin I-generating activity (data not shown), supporting that renin was the angiotensin Igenerating enzyme. The increase in angiotensin I-generating activity after prorenin activation (5.1 $\mathrm{ng}$ angiotensin $\mathrm{I} / \mathrm{ml} / \mathrm{h}$ ) corresponded to $20 \%$ to $30 \%$ of the angiotensin I-generating activity before prorenin stimulation (18.8 ng angiotensin I/ $\mathrm{ml} / \mathrm{h}$ ) (Figure $3 \mathrm{l}$ ). Juxtaglomerular cells are known to release mainly renin upon acute stimulation of cyclic adenosine monophosphate, and in further studies we therefore focused on renin only. Stimulation of the kidney organoids with forskolin resulted in a 20 -fold increase in intracellular renin activity compared with untreated cells (Figure $3 \mathrm{~m}$ ). The same rise in renin activity was observed in the conditioned culture medium (Figure 3n).

\section{Parathyroid hormone stimulates renin production in kidney organoids}

Next we sought to detect whether the renin-producing cells were responsive to hormonal stimuli. Initially we tested common regulators of renin to mimic signals from the macula densa to the juxtaglomerular apparatus. Organoids were stimulated with prostaglandins and cultured in low-salt conditions. No increase in renin was observed in these conditions (data not shown), most likely owing to the relative immaturity of the kidney organoids. Previous studies showed a relation between parathyroid hormone (PTH) stimulation and renin activity. ${ }^{15,16}$ We found that type $1 \mathrm{PTH}$ receptor (PTH1R) mRNA rises more than 100-fold during kidney organoid development (Figure $4 \mathrm{a}$ ). scRNAseq revealed that in a subset of stromal cells in the forskolin-treated organoid there was co-expression of renin and PTH1R (Figure 4b). A 20 -fold increase in renin mRNA in kidney organoids treated with $500 \mathrm{nM}$ PTH indicated a functional PTH1R (Figure 4c). Next we hypothesized that PTH could facilitate renin production and secretion by activation of its receptor. The phosphodiesterase inhibitor 3-isobutyl-1-methylxanthine (IBMX) was added to avoid degradation of cyclic adenosine monophosphate. Immunoreactivity of renin after stimulation with PTH or IBMX alone was present in a few stromal cells dispersed throughout the organoids, whereas the combination of the 2 factors culminated in a higher frequency of stromal cells positive for renin (Figure 4d). Forskolin-treated organoids were used as a positive control. Renin synthesis was evident by the 7 -fold increase in intracellular renin activity upon PTH stimulation, whereas stimulation with IBMX alone or in combination with PTH resulted in a 20 - and 25 -fold increase, respectively (Figure $4 \mathrm{e}$ ). We sought to determine whether this increase would result in secretion of renin. A trend could be observed in renin secretion by the addition of PTH (4-fold, not significant) or IBMX (15-fold) (Figure 4e). The combination of the 2 factors caused a significant 34 -fold rise of renin secretion into the supernatant (Figure 4e).

\section{The production of renin is maintained after subcutaneous transplantation of kidney organoids}

To improve maturation status, organoids were subcutaneously implanted in the flanks of immune-deficient mice and analyzed after 2 months. The organoids were invaded by surrounding vessels (Figure 5a). Vascular structures contained erythrocytes, confirming vascularization (Figure 5b), and were of human origin as witnessed by the presence of human mitochondria (Figure 5c). Tubular and glomerular structures were maintained as immunohistochemistry showed putative $\mathrm{WT}^{+}$glomeruli, Villin-1 ${ }^{+}$proximal tubuli, and $\mathrm{ECAD}^{+}$ distal tubuli (Figure $5 \mathrm{~d}$ ). Forskolin stimulation of organoid explants resulted in a 3 -fold increase in the excretion of renin compared with untreated explanted organoids (Figure 5e). Similar treatment of murine tissue served as a baseline control for the increase in renin activity. Immunostaining of renin in the explanted organoid revealed renin-positive cells near the juxtaglomerular localization (Figure 5f).

\section{DISCUSSION}

We successfully generated kidney organoids that recapitulate essential renal structures and explored the presence and 
a

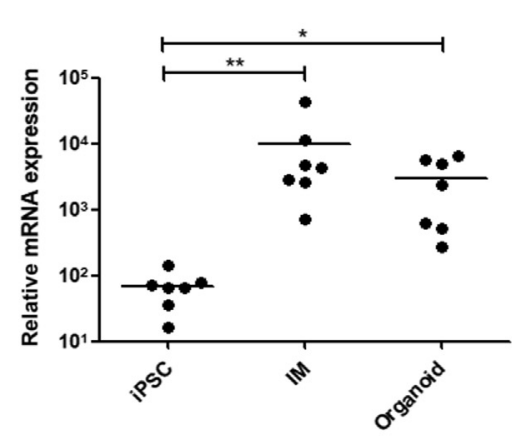

C

AGT

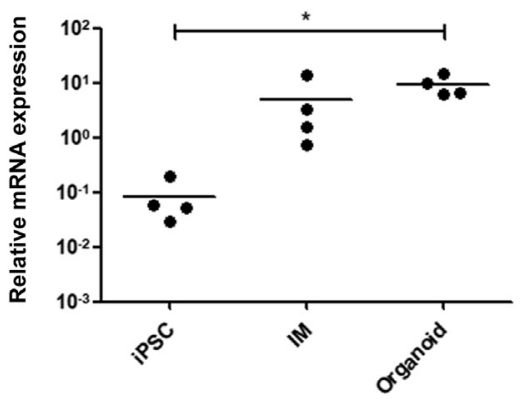

d

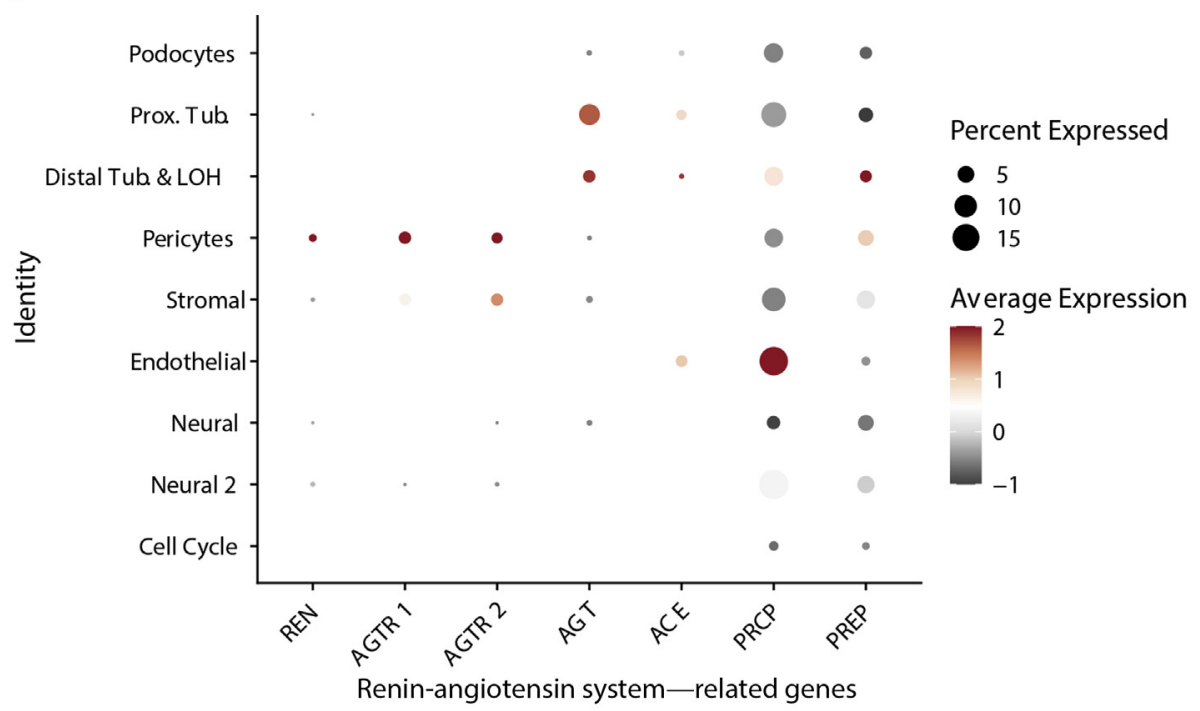

b

AGTR1

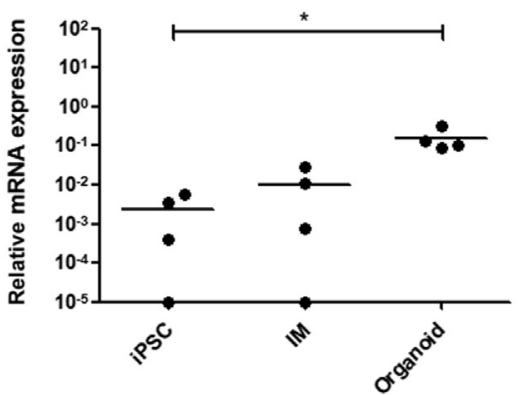

ACE

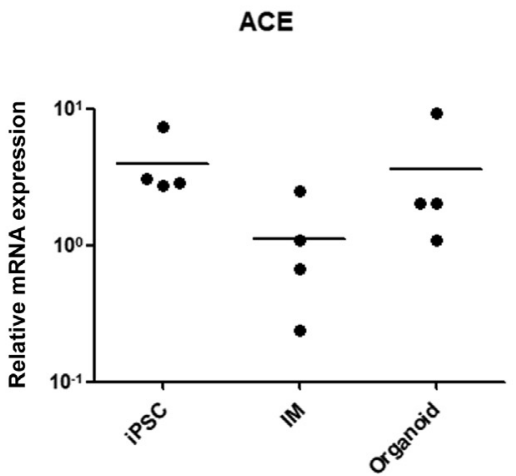

AGTR2

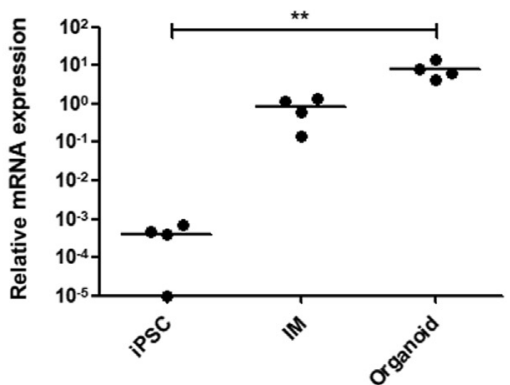

e

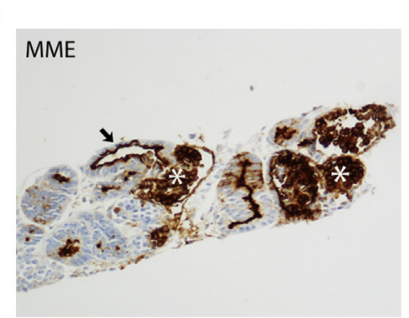

Figure 2 | Components of the RAS can be found in kidney organoids. (a) Gene expression analysis of renin over time at day 0 (iPSC), day 7 (IM), and day 25 (organoids) of the kidney organoid culture. Data are represented as mean \pm SEM ( $n=7$ from 3 independent experiments). Statistical analysis was performed using the Mann-Whitney test $\left({ }^{*} P<0.05\right.$; $\left.{ }^{* *} P<0.01\right)$. (b,c). Gene expression analysis of RAS components (AGTR1, AGTR2, AGT, and ACE) over time at day 0 (iPSC), day 7 (IM), and day 25 (organoids) of the kidney organoid culture. (Continued) 
function of the RAS, a fundamental kidney endocrine system. To our knowledge, this is the first study to show in a comprehensive and detailed manner that iPSC-derived human kidney organoids contain a unique population of reninproducing pericytes and that renin production is an inducible process. These results offer exciting opportunities for future in vitro and in vivo studies focused on the distribution and function of renin-producing cells in the human kidney, which has not been possible to date.

Kidney organoids do not contain vascularized glomeruli and the intricate signaling pathways between the macula densa and juxtaglomerular apparatus, as seen in the adult kidney. In addition, in the absence of glomerular filtration and tubular flow, NKCC2 may not sense sodium chloride, the starting point for renin release. In the current model it is therefore challenging to examine physiologic interactions with other important mediators of renin production. Previous studies have shown a direct stimulatory effect of PTH on renin secretion, but the exact mechanism underlying this relationship has not been elucidated yet. ${ }^{17-21}$ We show that PTH has a direct and strong effect on renin production.

To drive maturation, we implanted the organoids subcutaneously in mice, which led to vascularized renal structures. In this setting, a functional subset of renin-producing cells was maintained. The low levels of renin in implanted organoids may be due to fewer renin-positive cells after implantation, which is comparable to the adult kidney in which renin-producing cells constitute $0.01 \%$ of the cells. ${ }^{22}$ Furthermore, explanted organoids may not be exposed adequately to the forskolin stimulation owing to encapsulating cell layers. Novel approaches are being undertaken to improve maturation status through the use of microfluidic systems. ${ }^{1}$

Even though an increase in AGT mRNA was observed in the organoids, AGT protein was not detected in the supernatants. A recent publication demonstrated that liver-targeted AGT small interfering RNA decreases AGT and angiotensin I protein levels in the kidney without affecting renal $A G T$ mRNA expression. ${ }^{23}$ Renal angiotensin generation is therefore considered to depend on the uptake of hepatic angiotensinogen. The presence of AGTR1 and AGTR2 mRNA still suggests that the RAS contributes to the development of kidney organoids, yet we did not observe any effect of blocking either AGTR1 with losartan or renin with aliskiren. More research is necessary to fully elucidate the complex role of RAS in kidney organoid development.

Upon demonstrating the endocrine potential of kidney organoids, we expanded on their versatility for use in various applications. Our findings form a foundation to study the role of the RAS during kidney development, as kidney organoids roughly emulate this complex process in an in vitro setting. Moreover, kidney organoids can be used to model diseases such as chronic kidney disease and kidney fibrosis to study their contribution to the dysregulation of the RAS and novel RAS-targeting drugs may be evaluated in this model. Moreover, our insights contribute to further unraveling properties of renin-producing cells. Finally-and importantly_ these results support the potential application of human kidney organoids to restore the endocrine system of injured kidneys. In conclusion, the discovery of an endocrine system in the kidney organoid model presents a myriad of opportunities to study kidney development and disease, clinical therapies, and regeneration.

\section{METHODS}

\section{Derivation of human iPSCs}

Human primary skin fibroblasts from 5 healthy donors were reprogrammed to iPSCs as described earlier, using a single, multicistronic lentiviral vector encoding OCT4, SOX2, KLF4, and C$M Y C{ }^{24}$ In accordance with the medical ethics committee of the Erasmus University Medical Center (MEC-2017-248), written informed consent was obtained from the donors. ${ }^{25}$ All iPSC lines tested negative for Mycoplasma infection. The iPSC lines were cultured on irradiated mouse embryonic feeders before transition to a feeder-free culture system.

\section{Feeder-free culture of human iPSCs}

Frozen human iPSCs were thawed and cultured in Essential 8 medium (Thermo Fisher Scientific, Bleiswijk, The Netherlands) on Geltrex (Gibco, Waltham, MA). The medium was changed every day and cells passaged every 3 to 4 days in clumps, using $0.5 \mathrm{mM}$ ethylenediamine tetraacetic acid dissociation buffer (Gibco) at split ratios of $1: 4$ to $1: 8$. At the start of differentiation, iPSC single-cell suspensions were generated by TrypLE Select (Thermo Fisher Scientific) and seeded at a density of 25,000 viable cells $/ \mathrm{cm}^{2}$. RevitaCell Supplement (Thermo Fisher Scientific) was added the first 24 hours.

\section{Organoid differentiation}

The differentiation protocol was adapted from the work of Takasato et al. ${ }^{26}$. iPSCs were plated at 20,000 to $25,000 \mathrm{cells} / \mathrm{cm}^{2}$ in Essential 8 medium supplemented with RevitaCell. The next day, the medium was changed to STEMdiff APEL2 medium (APEL2) (STEMCELL Technologies, Vancouver, BC, Canada) supplemented with $8 \mu \mathrm{M}$ CHIR99021 (R\&D Systems, Minneapolis, MN), 5\% Protein Free Hybridoma Medium II (PFHMII, Thermo Fisher Scientific), and 1\% Antibiotic-Antimycotic (Thermo Fisher Scientific). After 3 to 4 days, the cells were stimulated with $200 \mathrm{ng} / \mathrm{ml}$ recombinant human fibroblast growth factor 9 (R\&D Systems) and $1 \mu \mathrm{g} / \mathrm{ml}$ heparin

Figure 2 | (Continued) Data are represented as mean \pm SEM ( $n=7$ from 3 independent experiments). Statistical analysis was performed using the Kruskal-Wallis test $\left({ }^{*} P<0.05 ;{ }^{* *} P<0.01\right)$. (d) Dot plot visualization to depict the expression of the RAS components per cluster. (e) Immunohistochemistry for markers of neprilysin (membrane metallo-endopeptidase $\left[\mathrm{MME}^{+}\right]$) were observed in the podocytes (white asterisk) and proximal tubuli (black arrow) and angiotensin-converting enzyme 2 (ACE2 ${ }^{+}$) positivity depicted in the tubular compartment (black arrow). Bars $=100 \mu \mathrm{m}$. Distal Tub. \& LOH, distal tubule and loop of Henle; IM, intermediate mesoderm; iPSC, induced pluripotent stem cells; Prox. Tub. proximal tubule; RAS, renin-angiotensin system; SEM, standard error of the mean. To optimize viewing of this image, please see the online version of this article at www.kidney-international.org. 
a

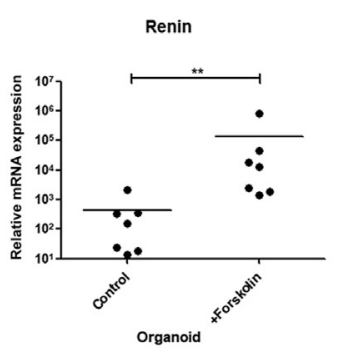

d

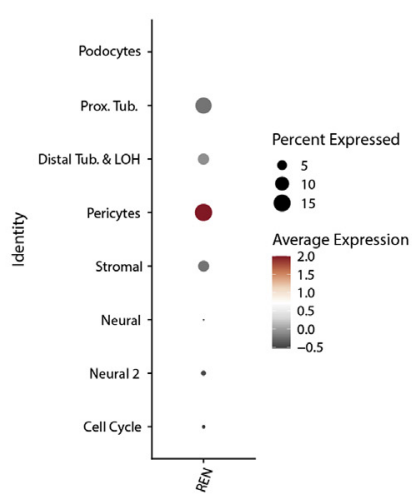

b

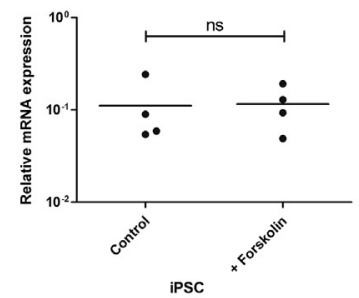

e

C RENIN

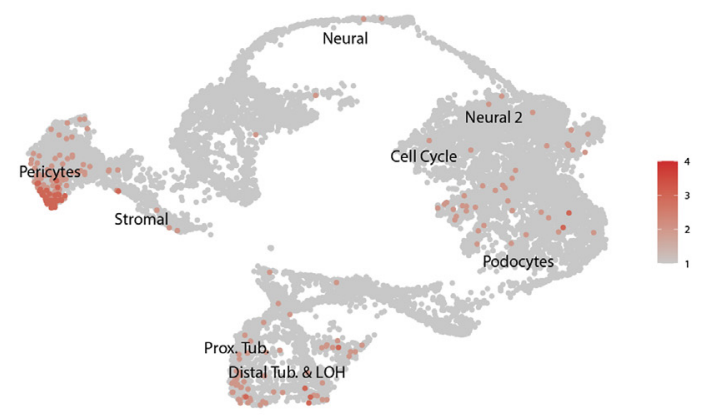

f

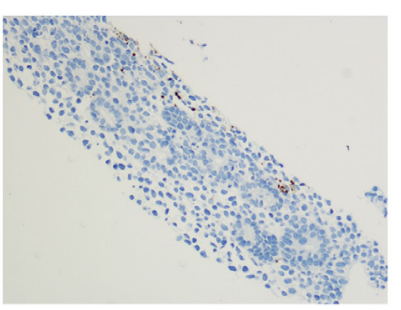

Control

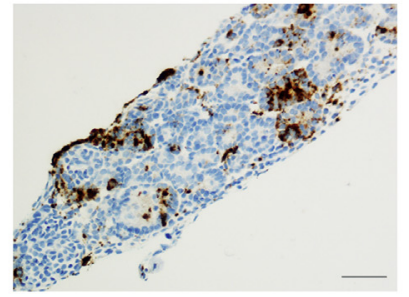

Forskolin g

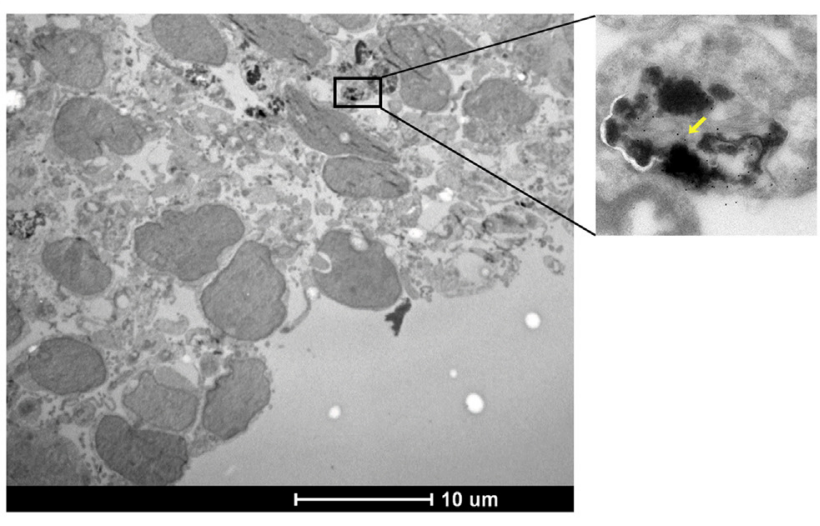

h
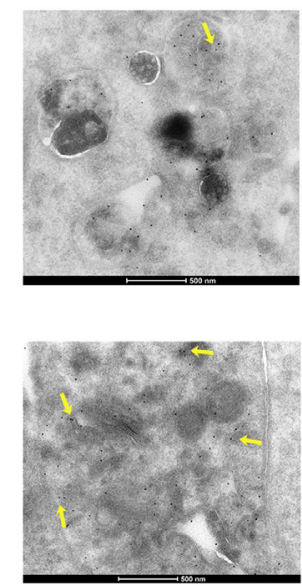

Figure 3 | Forskolin stimulates renin production in kidney organoids. (a) Comparison of renin gene expression levels in unstimulated and forskolin-stimulated kidney organoids. Data are represented as mean \pm SEM ( $n=8$ from 4 independent experiments). Statistical analysis was performed using the Mann-Whitney test $\left({ }^{* *} P<0.01\right)$. (b) Gene expression analysis for renin in iPSC after forskolin stimulation. Data are represented as mean \pm SEM ( $n=4$ from 1 experiment). Statistical analysis was performed using the Mann-Whitney test ( $n s$, nonsignificant). (c) Representation of uniform manifold approximation and projection with superimposed renin expression in forskolin-treated organoid. (d) Dot plot visualization of renin expression in the different clusters of forskolin-treated organoid. (e) Dot plot visualization of renin-related gene expression levels based on published literature in forskolin-treated organoid. (f) Immunohistochemical analysis for renin protein expression in control and forskolin-treated organoid. Bars $=100 \mu \mathrm{M}$. (g). Immunogold labeling of renin shows that the cytoplasm of potential pericytes contains granules with renin immunogold particles. The inset depicts multivesicular bodies from the late endocytic/lysosomal pathway. (h) Immunogold labeling in the cytosol (upper image) and in multivesicular bodies from the late endocytic/lysosomal pathway (lower image, yellow arrows). (Continued) 


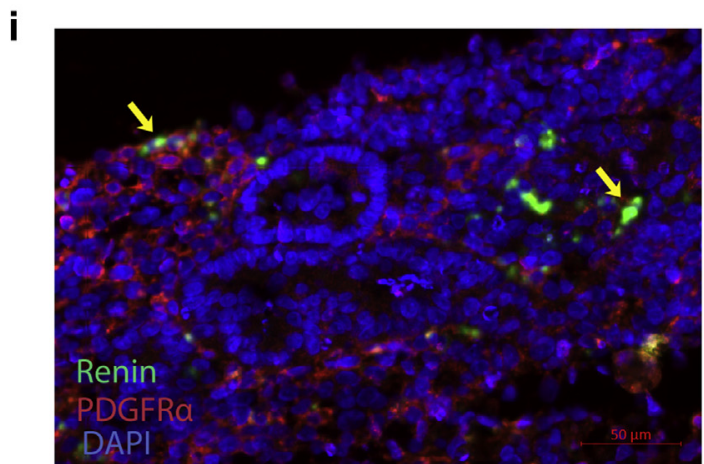

k

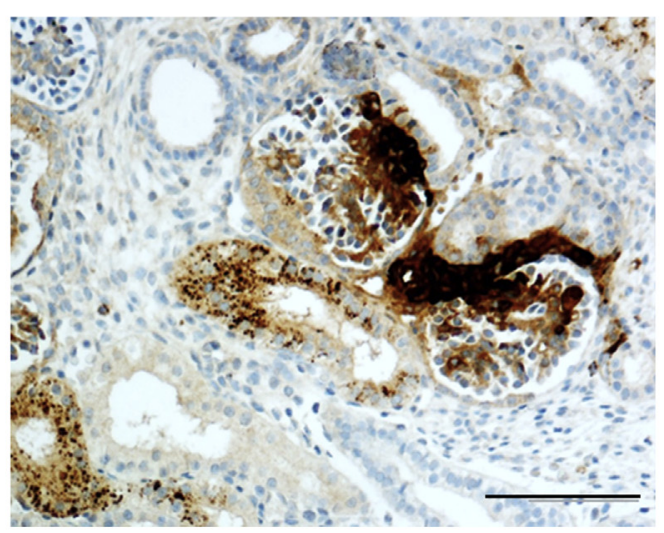

m

Renin in supernatant

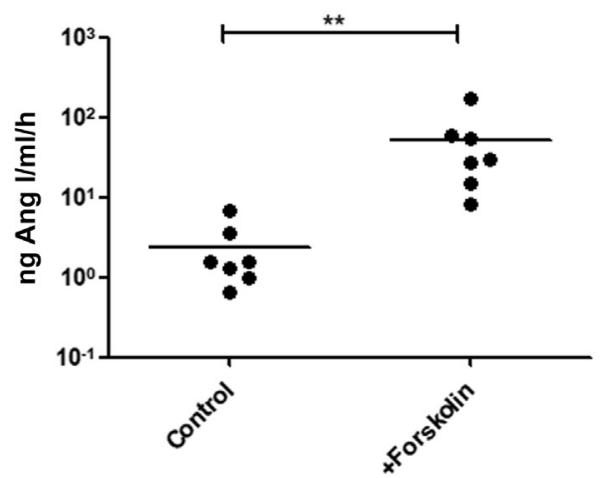

j

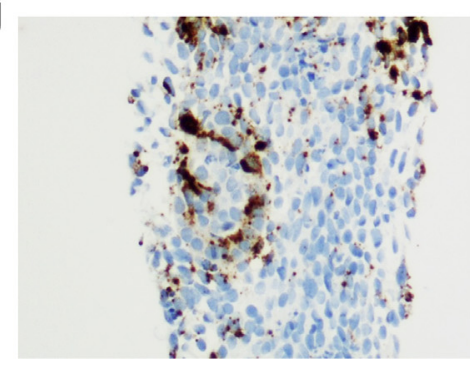

Renin

I

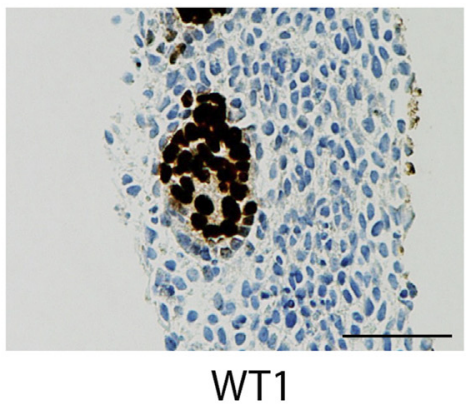

WT1

(Pro)renin in supernatant

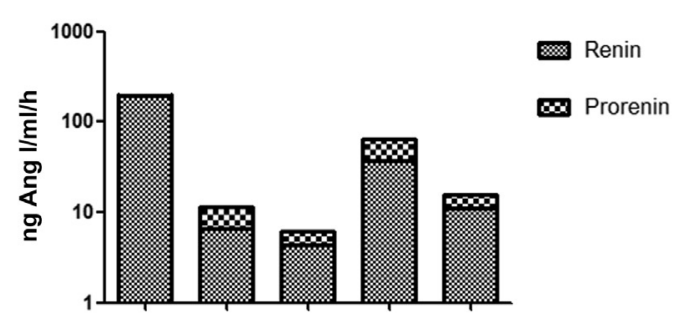

n

Intracellular renin

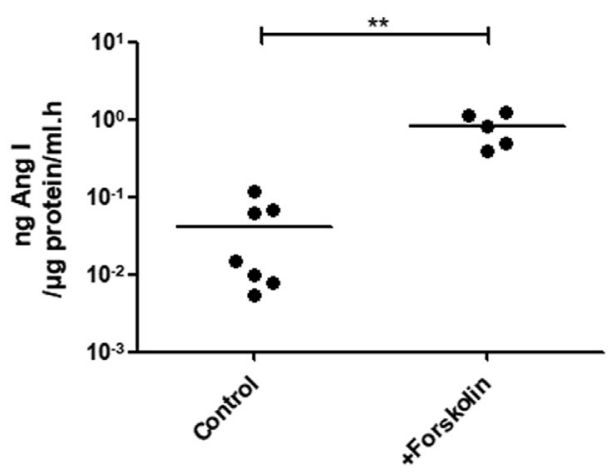

Figure 3 (Continued) (i) Double immunofluorescence staining for PDGFR $\alpha^{+}$(red) and renin positivity (green) showing colocalization (yellow arrows). Bar $=50 \mu \mathrm{M}$. (j) Immunohistochemical analysis for a marker of glomerular cells (WT1) and renin in consecutive slides. Bars $=100 \mu \mathrm{M}$. (k) Immunohistochemical analysis for renin in a 13-week-old fetal kidney. Bars $=100 \mu \mathrm{M}$. (I) Activity assay for prorenin and renin in the medium of 5 unstimulated organoids represented as $\mathrm{ng}$ Ang I (angiotensin I) $/ \mathrm{ml} / \mathrm{h}$. Each bar represents one organoid $(\mathrm{n}=5 \mathrm{from} 2$ independent experiments). (m,n) Activity assay for renin depicted as $n g$ Ang l/ $\mu \mathrm{g}$ protein/h in the cell lysates ( $\mathrm{n}=5$ from 3 independent experiments) and $\mathrm{ng} \mathrm{Ang} \mathrm{l} / \mathrm{ml} / \mathrm{h}$ in the supernatant after a 24-hour stimulation with forskolin ( $\mathrm{n}=7$ from 4 independent experiments). Data are represented as mean \pm SEM. Statistical analysis was performed using the Mann-Whitney test $\left.{ }^{* *} P<0.01\right)$. Distal Tub. \& LOH, distal tubule and loop of Henle; iPSC, induced pluripotent stem cells; PDGFR $\alpha$, platelet-derived growth factor receptor A; Prox. Tub., proximal tubule; SEM, standard error of the mean. To optimize viewing of this image, please see the online version of this article at www.kidney-international.org.

(Sigma-Aldrich, Zwijndrecht, The Netherlands) until day 7. Thereafter the cells were transferred to a 3-dimensional culture system in which 500,000 cells were placed on a Transwell filter membrane (0.4$\mu \mathrm{m}$ pore polyester membrane, Corning, Tewksbury, MA) exposed to air from above. The cells were pulsed for 1 hour with $5 \mu \mathrm{M}$ CHIR99021 and then stimulated with $200 \mathrm{ng} / \mathrm{ml}$ recombinant human fibroblast growth factor and $1 \mu \mathrm{g} / \mathrm{ml}$ heparin for 5 days. Thereafter growth factors were removed and the organoids maintained in APEL medium for 13 days and the medium was changed every other day. A total of 150 organoids from 5 lines were used in 7 experiments.

\section{Real-time qPCR}

mRNA was isolated using the High Pure RNA Isolation Kit (Roche Molecular Systems, South San Francisco, CA). Complement DNA was synthesized from $500 \mathrm{ng}$ mRNA with random primers (Promega 
a

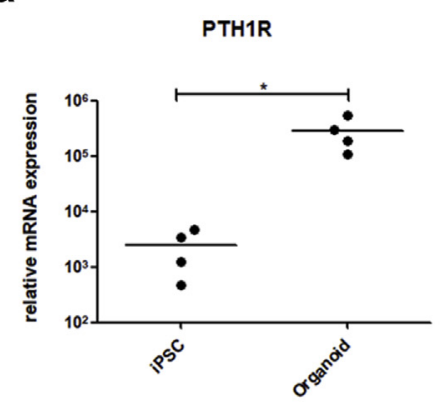

b

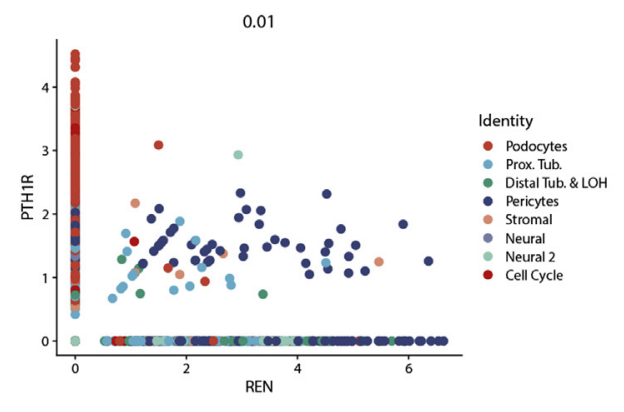

C

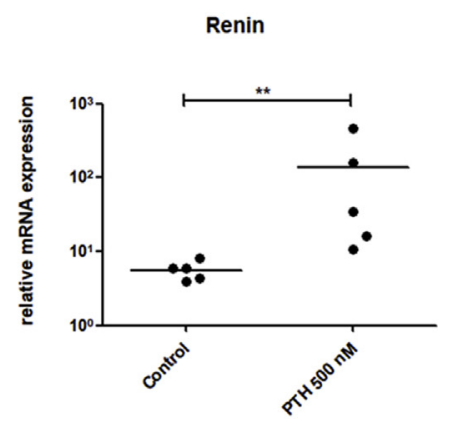

d

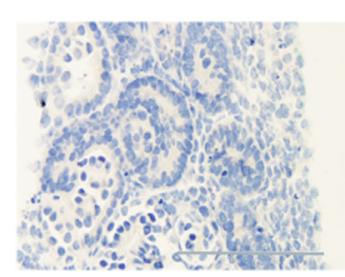

Control

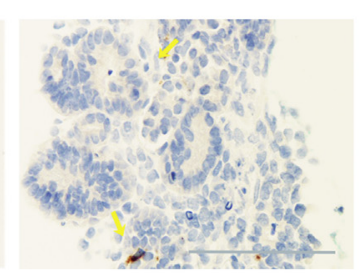

PTH

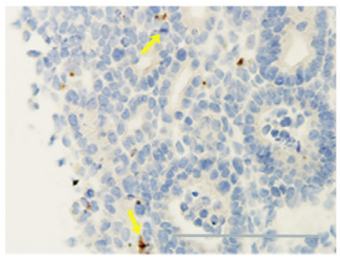

IBMX

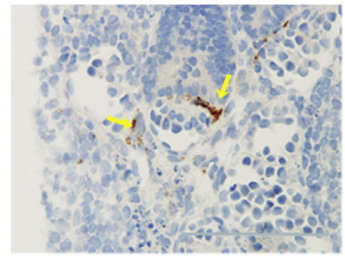

PTH + IBMX

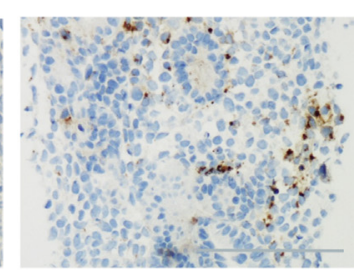

Forskolin

e
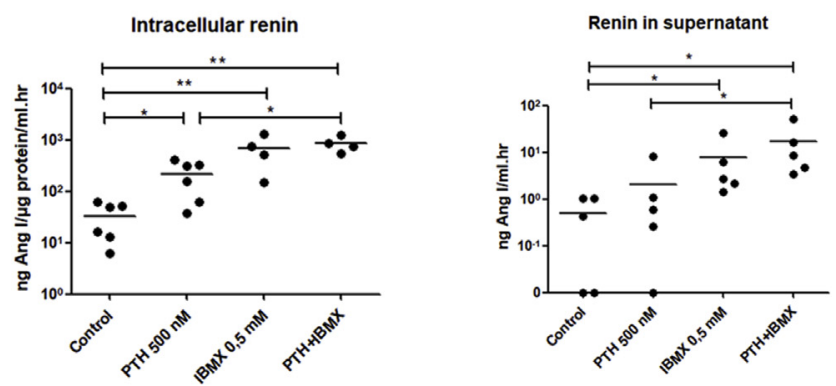

Figure 4 | Parathyroid hormone stimulates renin production in kidney organoids. (a) Gene expression analysis for type I parathyroid hormone receptor (PTH1R) in kidney organoids compared with undifferentiated iPSC. Data are represented as mean \pm SEM ( $n=4$ from 1 experiment). Statistical analysis was performed using the Mann-Whitney test $(P<0.05)$. (b) Scatter plot of PTHR1 and renin gene expression across cells in forskolin-treated organoid. Cells appear colored by their identity class. (c) Gene expression analysis for renin in organoids stimulated with PTH compared with control organoids. Data are represented as mean \pm SEM ( $n=5$ from 1 experiment). Statistical analysis was performed using the Mann-Whitney test $\left({ }^{*} P<0.01\right)$. (d) Immunohistochemical analysis of renin in organoids treated with PTH, IBMX, PTH + IBMX, and forskolin (positive control). The yellow arrows indicate the presence of renin-positive cells. Bars $=100 \mu \mathrm{M}$. (e) Activity assay for renin depicted as $\mathrm{ng}$ Ang $\mathrm{I} / \mu \mathrm{g}$ protein/h in the cell lysates of organoids treated with PTH, IBMX, and PTH + IBMX ( $\mathrm{n}=5$ from 1 experiment) and $\mathrm{ng}$ Ang $\mathrm{l} / \mathrm{ml} / \mathrm{h}$ in the supernatant ( $n=5$ from 1 experiment). Data are represented as mean $\pm \mathrm{SEM}$. Statistical analysis was performed using the Mann-Whitney test $\left({ }^{*} P<0.05 ;{ }^{* *} P<0.01\right)$. Distal Tub. \& LOH, distal tubule and loop of Henle; IBMX, 3-isobutyl-1-methylxanthine; iPSC, induced pluripotent stem cells; PDGFR $\alpha$, platelet-derived growth factor receptor A; Prox. Tub., proximal tubule; PTH, parathyroid hormone; SEM, standard error of the mean. To optimize viewing of this image, please see the online version of this article at www.kidney-international.org.

Benelux B.V., Leiden, The Netherlands). Expression levels were normalized to glyceraldehyde-3-phosphate dehydrogenase (Supplementary Figure S2). Quantitative gene expression was determined using TaqMan Gene Expression Assays-on-demand (Applied Biosystems, Foster City, CA) (Supplementary Table S2).

\section{Immunohistochemistry and immunofluorescence}

Four-micron sections of formalin-fixed paraffin embedded organoids were stained with hematoxylin and eosin (Ventana Medical Systems, Tucson, AZ). Immunohistochemistry was performed with an automated, validated, and accredited staining system (Ventana Benchmark ULTRA) using the UltraView or OptiView universal DAB detection Kit (Ventana Medical Systems). After deparaffinization and heat-induced antigen retrieval, the tissue samples were incubated with WT1 (Cell Marque, Rocklin, CA), ECAD (Ventana Medical Systems), Villin-1 (Abcam, Cambridge, UK), CD31 (Cell Marque), renin (Abcam), NKCC2 (StressMarq Biosciences, Victoria, BC, Canada), NHE3 (StressMarq), CD34 (Cell Marque), COL1A1 (Novus Biologicals, Littleton, CO), or PDGFR $\alpha$ (R\&D systems). Incubation was followed by hematoxylin II counterstaining. Double stainings of PDGFR $\alpha$ and renin were performed by incubating the samples with PDGFR $\alpha$ for 32 minutes at $37{ }^{\circ} \mathrm{C}$ followed by detection with Red610 kit (\#760-245, Ventana) and renin for 32 minutes at $37{ }^{\circ} \mathrm{C}$ followed by detection with FAM kit (\#760243, Ventana). An AF488-conjugated anti-human mitochondria antibody MAB1273A4 (Millipore, Billerica, MA) was used to detect 
a

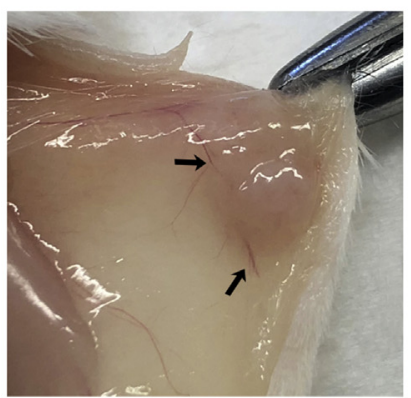

2 months after implantation

C
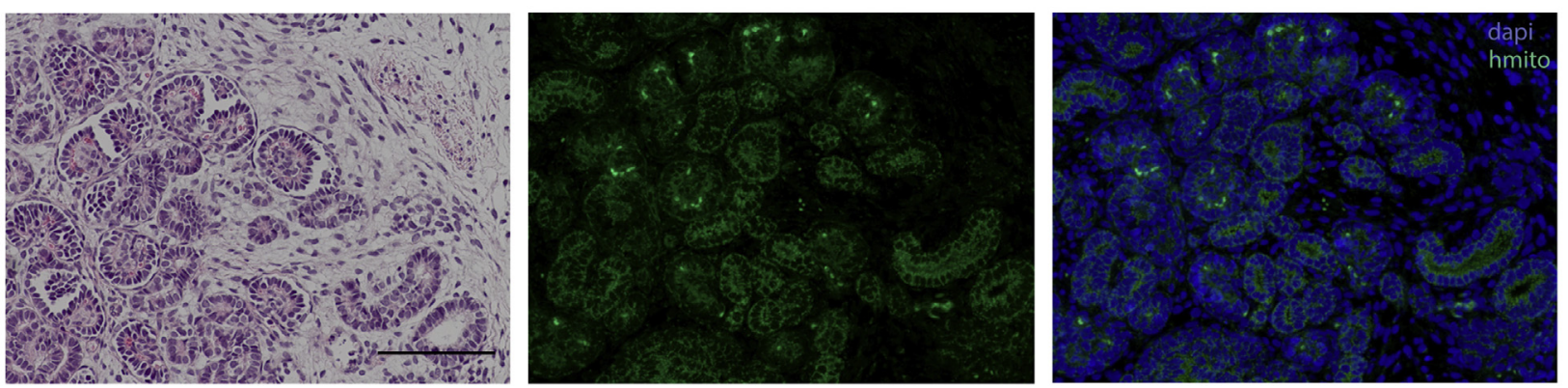

d

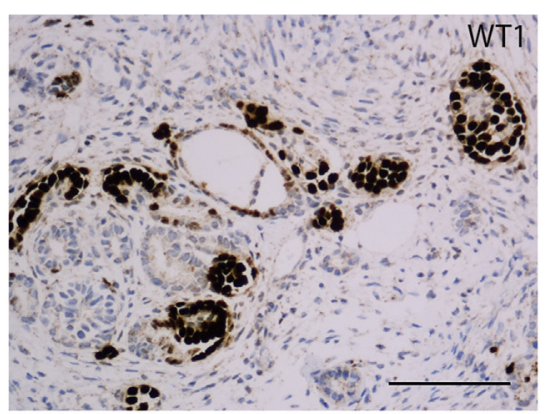

e

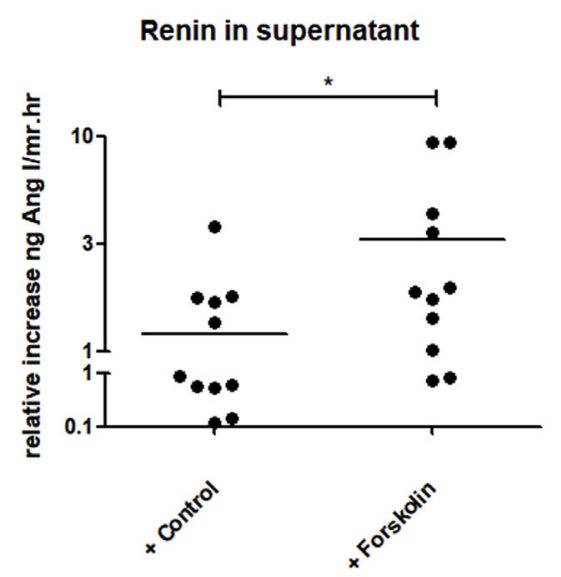

b

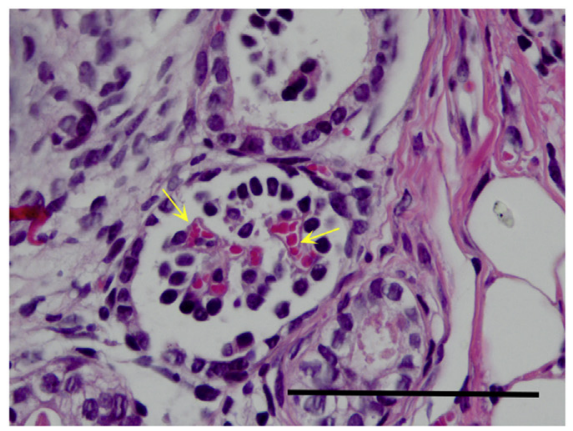

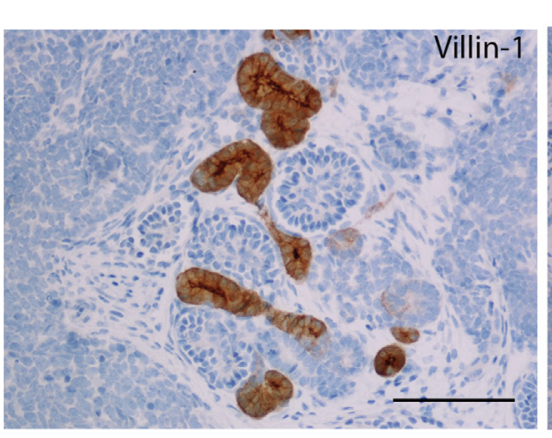

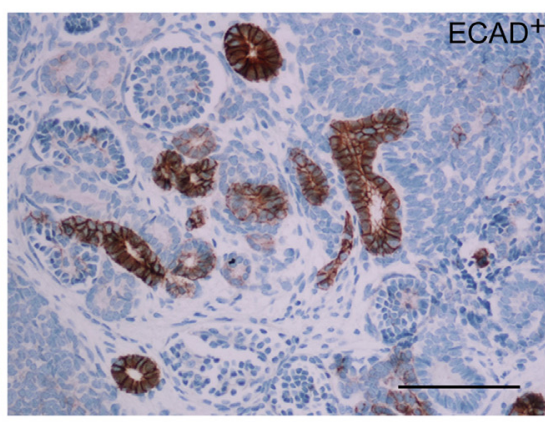

f

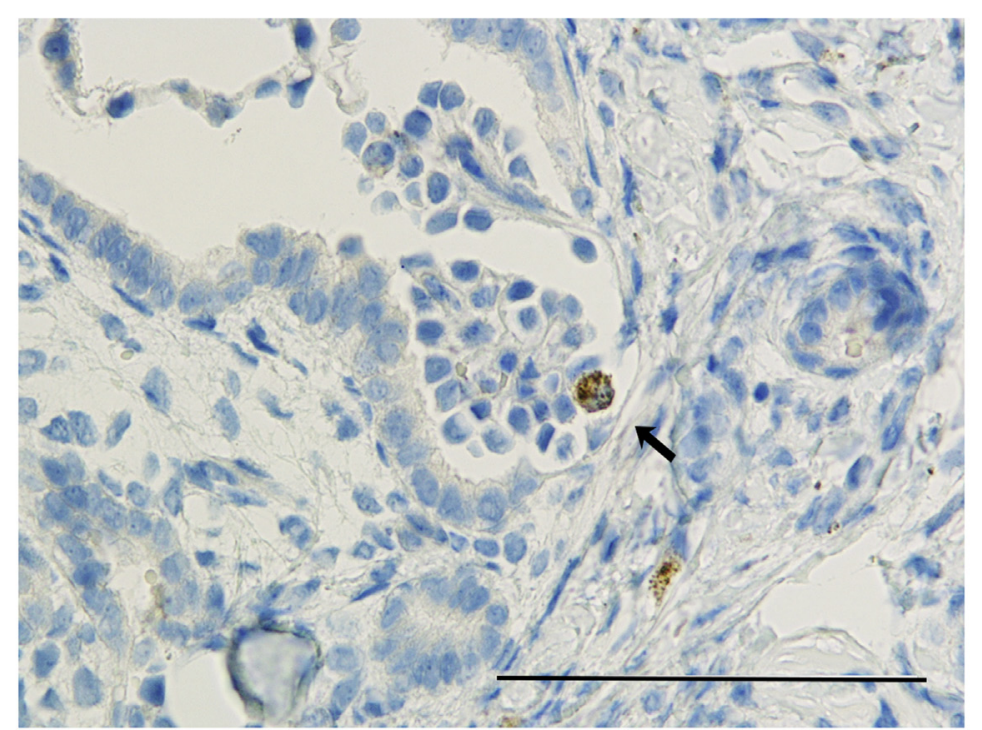

Figure 5 | The production of renin is maintained after subcutaneous transplantation of kidney organoids. (a) A photograph of a subcutaneously implanted kidney organoid in mice after 2 months. Black arrows indicate blood vessels migrating to the implanted organoid. (b) Histochemical staining (H\&E) of implanted structure containing erythrocytes. Arrows indicate the presence of erythrocytes. Bars $=50 \mu \mathrm{M}$. (c) H\&E of organoid after 2 months of implantation with visible tubular and glomerular structures (left). Double immunofluorescence (continued) 
human-specific tissue. Tonsil and healthy kidney tissue were used as positive controls. Fetal kidney was used in accordance with the Dutch laws on medical research and is regulated by the Netherlands Code of Conduct.

\section{Fluorescein uptake}

One $\mu \mathrm{M}$ fluorescein (Sigma-Aldrich) was added to kidney organoid culture medium for 1 hour at $37{ }^{\circ} \mathrm{C}$, as described previously. ${ }^{27} \mathrm{Ex}$ periments were performed on a Leica TCS SP5 confocal microscope (Leica Microsystems B.V., Eindhoven, The Netherlands). Images were processed using ImageJ version 1.48 software (National Institutes of Health, Washington, DC).

\section{Electron microscopy}

Organoids were fixed with $1.5 \%$ glutaraldehyde/0.067 M cacodylate $(\mathrm{pH} 7.4) / 1 \%$ sucrose. After 24 hours at $4{ }^{\circ} \mathrm{C}$, the samples were washed in $0.1 \mathrm{M}$ cacodylate buffer and fixed with $1 \%$ osmium tetroxide in the same buffer containing $1.5 \%$ potassium ferricyanide for 1 hour at $4{ }^{\circ} \mathrm{C}$. Subsequently, the samples were dehydrated, infiltrated with epoxy resin, and polymerized at $60{ }^{\circ} \mathrm{C}$ during 48 hours. Ultrathin sections were obtained using a Leica Ultracut UCT Ultramicrotome (Leica Microsystems). Samples were stained with $2 \%$ uranyl acetate in ethanol and lead citrate. Sections were imaged in a Tecnai T12 electron microscope equipped with an Eagle $4 \mathrm{kx} 4 \mathrm{k}$ CCD camera (Thermo Fisher Scientific).

\section{Immunogold labeling}

Ultrathin sections were prepared in an ultracryomicrotome (Leica EM Ultracut UC6/FC6, Leica Microsystems) and collected with 2\% methylcellulose in $2.3 \mathrm{M}$ sucrose. Cryosections were incubated on drops of $2 \%$ gelatin in phosphate-buffered saline (PBS) for $20 \mathrm{mi}$ nutes at $37{ }^{\circ} \mathrm{C}$, followed by $50 \mathrm{mM}$ glycine/PBS during 10 minutes and $1 \%$ bovine serum albumin (BSA)/PBS during 15 minutes. The samples were then incubated with anti-renin (Abcam) diluted 1:5 in $1 \%$ BSA/PBS for 60 minutes. Sections were incubated for 20 minutes with protein A coupled to $10-\mathrm{nm}$ diameter colloidal gold particles using a 1:50 dilution in 1\% BSA/PBS and observed by an Tecnai Spirit electron microscope (Thermo Fisher Scientific) with an Eagle $4 \mathrm{kx} 4 \mathrm{k}$ CCD camera.

\section{scRNAseq}

Single-cell suspensions of organoids were generated using TrypLE Select for 15 minutes at $37^{\circ} \mathrm{C}$ and stored on ice in APEL2 medium. Single-cell libraries were prepared using the Chromium Single Cell $3^{\prime}$ Reagent Kit v3 $(10 \times$ Genomics, Pleasanton, CA). Next-generation sequencing (28-8-0-91 cycles) was performed on an Illumina NovaSeq 6000 platform (Illumina, San Diego, CA). Raw data were processed into FASTQ files. Raw sequences were inspected for their quality using FastQC (version 0.11.5, Babraham Bioinformatics, Cambridge UK). Readings containing sequence information were mapped to the GRCh38 human reference genome. Generation of BAM files and filtered gene-barcode matrices was accomplished using the Cell Ranger Software (version 3.0.2, 10X Genomics, Pleasanton, CA).

Analysis. Analysis was done using Seurat software. ${ }^{28}$ Initial filtering removed genes that were expressed in fewer than 3 cells and cells with fewer than 200 distinct transcripts. In addition, mitochondrial and ribosomal genes were filtered out. Data were normalized for sequencing depth by dividing by the total number of unique molecular identifiers in every cell and then transformed to a $\log$ scale for each cell using the NormalizeData function. Normalized data were scaled to the mean across cells and SD of 1 using the ScaleData function. Principal component analysis was performed on the 10,000 most variable genes; 15 principal components were significant after reaching 2 SDs of the principal components. Cells were then clustered using the shared nearestneighbor modularity optimization-based clustering algorithm implemented in Seurat using the first 15 principal components and a resolution of 0.5 . Uniform manifold approximation and projection (UMAP) was used for the 2-dimensional representation of the data using the RunUMAP function.

Marker gene lists were generated to find differentially expressed genes between clusters, with a log-fold change above 0.25 . Annotation of clusters to cell types was done by manual inspection of the features defining each cluster and comparison to previous studies. ${ }^{29-32}$ Visualizations using the DotPlot function graphically represent the percentage of expression per cluster by dot diameter and average nonzero expression in $\log 2$ scale by dot color; data were not shown if the percentage of expressing cells was less than 0.01 .

\section{Renin and angiotensinogen measurements}

Renin and total renin (renin + prorenin) were measured with an enzyme kinetic assay measuring the conversion of angiotensinogen to angiotensin I. ${ }^{33}$ Prorenin was calculated by subtracting renin from total renin. One ng angiotensin $\mathrm{I} / \mathrm{ml} / \mathrm{h}$ corresponds to $2.6 \mathrm{pg}$ human renin $/ \mathrm{ml}^{33}$ The direct renin inhibitor aliskiren $(10 \mu \mathrm{M})$ was used to block angiotensin I-generating activity. ${ }^{34}$ Angiotensinogen was measured after its conversion to angiotensin I by excess renin. Angiotensin I was measured by radioimmunoassay.

\section{Functional experiments}

Five hundred nM angiotensin II (Sigma-Aldrich) with or without 10 $\mu \mathrm{M}$ losartan (Sigma-Aldrich), or $1 \mu \mathrm{M}$ aliskiren (a gift from Novartis Pharmaceuticals, Basel, Switzerland) was added from day 12 until day 25 and samples were collected for RNA extraction and immunohistochemistry.

Stimulation of intracellular cyclic adenosine monophosphate To elevate intracellular cyclic adenosine monophosphate, $10 \mu \mathrm{M}$ forskolin (STEMCELL Technologies) or $500 \mathrm{nM}$ PTH 1-84 (ProSpec, East Brunswick, NJ) alone or in combination with phosphodiesterase inhibitor IBMX (Sigma-Aldrich) was added to the culture medium

Figure 5 | (continued) analysis for a human-specific marker (human mitochondria) and a nuclear marker (4',6-diamidino-2-phenylindole [DAPI]) of consecutive slide. Bars $=50 \mu \mathrm{M}$. (d) Immunohistochemical staining for glomerular structures (WT1), proximal tubular structures $\left(\right.$ Villin-1), and distal tubular structures $\left(E-c a d h e r i n ~[E C A D]^{+}\right)$. Bars $=100 \mu \mathrm{M}$. (e) Activity assay for renin measurement in the medium of explanted kidney organoids after 24-hour forskolin treatment, depicted as ng Ang l/ml/h. Data are represented as mean \pm SEM $(n=11$ from 2 independent experiments). Statistical analysis was performed using the Mann-Whitney test $\left({ }^{*} P<0.05\right)$. (f) Immunohistochemical staining for renin in explanted kidney organoid after forskolin treatment (black arrow). Bars $=100 \mu \mathrm{M}$. To optimize viewing of this image, please see the online version of this article at www.kidney-international.org. 
for 24 hours, after which kidney organoids were harvested for gene expression analysis, renin activity assay, and immunohistochemistry.

\section{Subcutaneous implantation of kidney organoids}

Subcutaneous implantation of kidney organoids was performed in immune-deficient mice (BALB/c Il $2 \mathrm{Ry}^{-1-} / \mathrm{Rag}^{-{ }^{-1}}$ double knockout), which lack lymphocytes. The organoids were inserted in $50 \mu \mathrm{l}$ Geltrex in 4 dorsal pockets. Four and 8 weeks after surgery, the animals were euthanized and the implanted organoids were isolated. The explanted organoids were ex vivo cultured or fixed in $4 \%$ paraformaldehyde or frozen at $-80{ }^{\circ} \mathrm{C}$ for immunohistochemistry and mRNA gene expression analysis. Explanted organoids were cultured with $10 \mu \mathrm{M}$ forskolin-containing APEL2 medium for 24 hours and the medium was collected for renin activity assay. The experiments were approved by the Animal Ethical Committee of the Erasmus Medical Center (licence number AVD101002016635).

\section{Statistics}

The Mann-Whitney test and the Kruskal-Wallis test with the Dunn post hoc test were used for comparisons between 2 or more than 2 groups, respectively. Data are expressed as the mean plus or minus the standard error of the mean. Statistical significance was considered for $P$ values less than $0.05\left(^{*}\right)$ and $0.01\left(^{* *}\right)$. Data analysis was performed using GraphPad Prism (GraphPad Software, La Jolla, CA).

\section{DISCLOSURE}

All the authors declared no competing interests.

\section{ACKNOWLEDGMENTS}

We thank the Microscopy CORE Lab team of FHML Maastricht University, specifically Willine van de Wetering, for the support in the Electron Microscopy experiments. We also thank the colleagues at the department of Pathology, Erasmus Medical Center Rotterdam, for their support in processing samples. This study was supported by the MRACE Erasmus MC grant 2015.

\section{AUTHOR CONTRIBUTIONS}

ASS designed and performed experiments, analyzed results and wrote the manuscript. ZD, TPPvdB, SSK, and IMVdB-G performed experiments. HTM analyzed results. EB, CL-I, MCC-vG, JG, and CCB provided expertise and reviewed the manuscript. AHJD, EJH, and MJH designed the experiments, provided expertise and feedback, and reviewed the manuscript.

\section{SUPPLEMENTARY MATERIAL}

Supplementary File (PDF)

Figure S1. (A) Gene expression analysis of RAS components (AGTR1, AGTR2, $A G T$, and renin) in day-25 kidney organoids after treatment with $500 \mathrm{nM}$ angiotensin II $\pm 10 \mu \mathrm{M}$ losartan or with $1 \mu \mathrm{M}$ aliskiren $(\mathrm{n}=2)$. (B) Gene expression analysis of pro-fibrotic markers SERPIN E1 and TGF-beta in day-25 kidney organoids after treatment with $500 \mathrm{nM}$ angiotensin II $\pm 10 \mu \mathrm{M}$ losartan or with $1 \mu \mathrm{M}$ aliskiren $(n=2)$. (C) Gene expression analysis of differentiation markers WT1, Villin-1, and ECAD in day-25 kidney organoids after treatment with $500 \mathrm{nM}$ angiotensin II $\pm 10 \mu \mathrm{M}$ losartan or with $1 \mu \mathrm{M}$ aliskiren $(\mathrm{n}=2)$. (D) Immunohistochemical analysis for markers of fibrosis (COL1A1), glomerular cells (WT1), and proximal tubular cells (Villin-1). Bars $=200 \mu \mathrm{m}$.

Figure S2. Gene expression analysis of housekeeping genes across organoids showing low variation between samples.

Table S1. Contribution of each cell population expressed as the number and percentage of cells per cluster.

Table S2. List of primers used for analyzed genes.

\section{REFERENCES}

1. Homan KA, Gupta N, Kroll KT, et al. Flow-enhanced vascularization and maturation of kidney organoids in vitro. Nat Methods. 2019;16: 255-262.

2. Takasato $\mathrm{M}$, Little MH. Making a kidney organoid using the directed differentiation of human pluripotent stem cells. Methods Mol Biol. 2017;1597:195-206.

3. Tanigawa $S$, Islam M, Sharmin $S$, et al. Organoids from nephrotic disease-derived iPSCs identify impaired NEPHRIN localization and slit diaphragm formation in kidney podocytes. Stem Cell Reports. 2018;11: 727-740.

4. Kurtz A. Endocrine functions of the renal interstitium. Pflugers Arch. 2017;469:869-876.

5. Shaw I, Rider S, Mullins J, et al. Pericytes in the renal vasculature: roles in health and disease. Nat Rev Nephrol. 2018;14:521-534.

6. Sequeira-Lopez ML, Lin EE, Li M, et al. The earliest metanephric arteriolar progenitors and their role in kidney vascular development. Am J Physiol Regul Integr Comp Physiol. 2015;308:R138-R149.

7. Stefanska A, Kenyon C, Christian HC, et al. Human kidney pericytes produce renin. Kidney Int. 2016;90:1251-1261.

8. Castrop H, Hocherl K, Kurtz A, Schweda F, et al. Physiology of kidney renin. Physiol Rev. 2010;90:607-673.

9. Brunskill EW, Sequeira-Lopez ML, Pentz ES, et al. Genes that confer the identity of the renin cell. J Am Soc Nephrol. 2011;22:2213-2225.

10. Monteil V, Kwon H, Prado P, et al. Inhibition of SARS-CoV-2 infections in engineered human tissues using clinical-grade soluble human ACE2. Cell. 2020;181:905-913.e907.

11. Mezzano SA, Ruiz-Ortega M, Egido J. Angiotensin II and renal fibrosis Hypertension. 2001;38(3 Pt 2):635-638.

12. Martini AG, Xa LK, Lacombe MJ, et al. Transcriptome analysis of human reninomas as an approach to understanding juxtaglomerular cell biology. Hypertension. 2017;69:1145-1155.

13. Sequeira Lopez ML, Pentz ES, Nomasa T, et al. Renin cells are precursors for multiple cell types that switch to the renin phenotype when homeostasis is threatened. Dev Cell. 2004;6:719-728.

14. Peters PJ, Borst J, Oorschot V, et al. Cytotoxic T lymphocyte granules are secretory lysosomes, containing both perforin and granzymes. J Exp Med. 1991;173:1099-1109.

15. Saussine C, Judes C, Massfelder T, et al. Stimulatory action of parathyroid hormone on renin secretion in vitro: a study using isolated rat kidney, isolated rabbit glomeruli and superfused dispersed rat juxtaglomerular cells. Clin Sci (Lond). 1993;84:11-19.

16. Smith JM, Mouw DR, Vander AJ. Effect of parathyroid hormone on plasma renin activity and sodium excretion. Am J Physiol. 1979;236:F311F319.

17. Saussine C, Massfelder T, Parnin F, et al. Renin stimulating properties of parathyroid hormone-related peptide in the isolated perfused rat kidney. Kidney Int. 1993;44:764-773.

18. Grant FD, Mandel SJ, Brown EM, et al. Interrelationships between the renin-angiotensin-aldosterone and calcium homeostatic systems. J Clin Endocrinol Metab. 1992;75:988-992.

19. Helwig JJ, Musso MJ, Judes C, Nickols GA. Parathyroid hormone and calcium: interactions in the control of renin secretion in the isolated, nonfiltering rat kidney. Endocrinology. 1991;129:1233-1242.

20. Broulik PD, Horky K, Pacovsky V. Effect of parathyroid hormone on plasma renin activity in humans. Horm Metab Res. 1986;18:490-492.

21. Vaidya A, Brown JM, Williams JS. The renin-angiotensin-aldosterone system and calcium-regulatory hormones. J Hum Hypertens. 2015;29: 515-521.

22. Gomez RA, Sequeira-Lopez ML. Novel functions of renin precursors in homeostasis and disease. Physiology (Bethesda). 2016;31:25-33.

23. Uijl E, Mirabito Colafella KM, Sun Y, et al. Strong and sustained antihypertensive effect of small interfering RNA targeting liver angiotensinogen. Hypertension. 2019;73:1249-1257.

24. Warlich E, Kuehle J, Cantz T, et al. Lentiviral vector design and imaging approaches to visualize the early stages of cellular reprogramming. Mol Ther. 2011;19:782-789.

25. de Esch CE, Ghazvini M, Loos F, et al. Epigenetic characterization of the FMR1 promoter in induced pluripotent stem cells from human fibroblasts carrying an unmethylated full mutation. Stem Cell Reports. 2014;3:548-555.

26. Takasato M, Er PX, Chiu HS, Little MH. Generation of kidney organoids from human pluripotent stem cells. Nat Protoc. 2016;11: 1681-1692. 
27. Jansen J, Fedecostante M, Wilmer MJ, et al. Bioengineered kidney tubules efficiently excrete uremic toxins. Sci Rep. 2016;6:26715.

28. Butler A, Hoffman $P$, Smibert $P$, et al. Integrating single-cell transcriptomic data across different conditions, technologies, and species. Nat Biotechnol. 2018;36:411-420.

29. Wu H, Uchimura K, Donnelly EL, et al. Comparative analysis and refinement of human psc-derived kidney organoid differentiation with single-cell transcriptomics. Cell Stem Cell. 2018;23:869-881. e868.

30. Phipson B, Er PX, Combes AN, et al. Evaluation of variability in human kidney organoids. Nat Methods. 2019;16:79-87.
31. Combes AN, Zappia L, Er PX, et al. Single-cell analysis reveals congruence between kidney organoids and human fetal kidney. Genome Med. 2019;11:3.

32. Garreta E, Prado P, Tarantino C, et al. Fine tuning the extracellular environment accelerates the derivation of kidney organoids from human pluripotent stem cells. Nat Mater. 2019;18:397-405.

33. Krop M, Garrelds IM, de Bruin RJ, et al. Aliskiren accumulates in renin secretory granules and binds plasma prorenin. Hypertension. 2008;52: 1076-1083.

34. Batenburg WW, Danser AJ. Prorenin and the (pro)renin receptor: binding kinetics, signalling and interaction with aliskiren. J Renin Angiotensin Aldosterone Syst. 2008;9:181-184. 